Methods 400 addicts were recruited from methadone clinics in 2009-2010. A self-designed questionnaire with excellent reliability was used to determine those with and without high risk behaviours. ORs with $95 \%$ CIs were estimated by logistic regression. Ethics approval was obtained from Tehran University.

Results There was significant difference between the two study groups (with and without high risk behaviours) in economic status, drug type, administration route, age, and drug abuse onset age. Education level was significantly lower in women. One quarter experienced homeless, $62 \%$ had no support from any insurance or supportive organisations, only $26 \%$ had constant employment and over $50 \%$ had prison history. $12.5 \%$ were injection users and $14 \%$ shared syringes. One quarter reported high risk sexual behaviour and $69.4 \%$ had not used condoms in last their last sex encounter (significantly lower in women). A decrease of one year in age was associated with decreased drug use onset age and increased sexual risk behaviours by $6 \%(\mathrm{AOR}=0.94,95 \% \mathrm{CI} 0.91$ to 0.98$)$ and $10 \%$ (AOR $=0.91,95 \%$ CI 0.85 to 0.97 ) respectively. Poor economic status reduced risk sexual behaviour ( $\mathrm{AOR}=0.35,95 \%$ CI 0.13 to 0.96 ). Prison history increased injection behaviour more than twice (AOR $=2.89,95 \%$ CI 1.4 to 5.95 )

Conclusions These findings illustrate that interventions are needed in young heroin users even in those with a good economic state.

\section{P2-258 EVALUATION OF THE EFFECTIVENESS OF THE PHARMACOTHERAPY FOLLOW-UP ON THE TREATMENT OF HYPERTENSIVE PATIENTS: A COHORT STUDY}

\section{doi:10.1136/jech.2011.142976j.91}

${ }^{1} \mathrm{~F}$ Rodrigues, ${ }^{2,3} \mathrm{M}$ Castro, ${ }^{1,2} \mathrm{~S}$ Fuchs, ${ }^{1,2}$ F Fuchs, ${ }^{1,2}$ L Moreira. ${ }^{* 1}$ Postgraduate Studies Program in Health Sciences: Cardiology and Cardiovascular Sciences; UFRGS, Porto Alegre, Rio Grande do Sul, Brazil; ${ }^{2}$ Division of Cardiology, Hospital de Clínicas de Porto Alegre, Porto Alegre, Rio Grande do Sul, Brazil; ${ }^{3}$ Pharmacy School, UFRGS, Porto Alegre, Rio Grande do Sul, Brazil

Introduction The control rate of hypertension in the population is fair. Pharmaceutical Care is a recent approach, seeking to magnify therapeutic results.

Objective To evaluate the effectiveness of the pharmacotherapy follow-up conducted by the Pharmacist on hypertension management.

Methods The study is a historical cohort with a dynamic population of patients referred to an outpatient hypertension clinic. Patients were followed for 12 months. Those difficult-to-control referred by the physician to pharmacotherapy follow-up were compared with patients under conventional treatment. Endpoints included blood pressure (BP) variation and the rate of control $(<140 / 90 \mathrm{~mm} \mathrm{Hg})$. General Linear Model, modified Poisson Regression, and segmented regression were used in the data analysis.

Results Of 993 patients, 150 were referred for pharmacotherapy follow-up. Patients in the pharmacotherapy follow-up were older, with lower education level, longer diagnoses of hypertension and significantly higher levels of BP. The deltas of SBP were $7.4 \pm 1.9$ vs $10.3 \pm 0.8 \mathrm{~mm} \mathrm{Hg} \quad(p=0.16)$ and diastolic $6.7 \pm 1.0 \mathrm{~mm} \mathrm{Hg}$ vs $5.9 \pm 0.4(p=0.45)$ for pharmacotherapy follow-up and conventional treatment groups, respectively, adjusted for initial pressure. The control rate was $45.3 \%$, being $28.1 \%$ in the exposed and $48.6 \%$ in the unexposed $(p<0.001)$. Comparing the BP of the same patients before and after exposure to pharmacotherapy follow-up showed a change in the trend of SBP and DBP $(p<0.001)$.

Conclusion Hypertensive patient difficult-to-control under pharmacotherapy follow-up showed a reduction in BP similar to patients who received only conventional treatment. The study suggests that pharmacotherapy follow-up is effective in the management of selected hypertensive patients.

\section{P2-259 CANCER RISK IN CHILDREN WITH BIRTH DEFECTS: A LONGITUDINAL, POPULATION-BASED ASSESSMENT AMONG 2.7 MILLION BIRTHS}

doi:10.1136/jech.2011.142976j.92

${ }^{1} \mathrm{P}$ A Romitti, ${ }^{2} \mathrm{~T}$ Flood, ${ }^{3} \mathrm{M}$ L Feldkamp, ${ }^{3} \mathrm{~S}$ Krikov, ${ }^{1} \mathrm{~S}$ Puzhankara, ${ }^{1} \mathrm{R}$ Goedken, ${ }^{3} \mathrm{M}$ Fluchel, ${ }^{4} \mathrm{~J}$ Little, ${ }^{3} \mathrm{~L} \mathrm{D}$ Botto. ${ }^{1}$ The University of lowa, lowa City, lowa, USA; ${ }^{2}$ Arizona Department of Public Health, Phoenix, Arizona, USA; ${ }^{3}$ University of Utah, Salt Lake City, Utah, USA; ${ }^{4}$ University of Ottawa, Ottawa, Ontario, Canada

Introduction The published literature, to date, is largely inconclusive regarding cancer risk among children with birth defects. To improve knowledge of such risk, we studied population-based (statewide) birth cohorts from Arizona, Iowa, and Utah selected from among 2.7 million births delivered from 1983 to 2006

Methods Birth defect and cancer diagnoses were identified from linked population-based surveillance systems. A population-based cohort of over 43000 children with major birth defects (including trisomies 13, 18, and 21) was compared to a cohort of nearly 148000 births without birth defects, randomly sampled from the same underlying birth population and frequency-matched to the birth defects cohort by birth year. Kaplan-Meier time-to-event analysis, accounting for censoring by death, was used to estimate cancer risk up to age 15 years.

Results Compared to the reference cohort, children with birth defects had a statistically significant increase in cancer risk ( $R R$, 2.73). Risk was highest among children with Down syndrome (RR, 13.2), and was driven largely by leukaemias. Cancer risk was moderately increased among children with a birth defect but without chromosomal anomalies (RR, 1.82). In this group, cancer risk was driven largely by brain tumours and embryonal tumours, and occurred mainly in children with brain defects, cleft palate, rectal defects, and some heart defects.

Conclusion These population-based findings support and extend previous findings that suggest increased cancer risk in children with birth defects, including non-chromosomal defects, and suggest selected defect groups in which further research could help identify a common genetic susceptibility to cancer and birth defects.

\section{P2-260 POPULATION-BASED PREVALENCE OF DUCHENNE/BECKER MUSCULAR DYSTROPHY (DBMD) IN THE USA}

doi:10.1136/jech.2011.142976j.93

${ }^{1} \mathrm{P}$ Romitti, ${ }^{1} \mathrm{~S}$ Puzhankara, ${ }^{1} \mathrm{G}$ Zamba, ${ }^{1} \mathrm{~S}$ Nabukera, ${ }^{5} \mathrm{~K}$ James, ${ }^{3} \mathrm{~J}$ Andrews, ${ }^{2} \mathrm{D}$ Fox, ${ }^{3} \mathrm{C}$ Cunniff, ${ }^{4} \mathrm{E}$ Ciafaloni, ${ }^{2} \mathrm{C}$ Druschel, ${ }^{1} \mathrm{~K}$ Mathews, ${ }^{5} \mathrm{D}$ Matthews, ${ }^{6} \mathrm{~L}$ Miller, ${ }^{4} \mathrm{~S}$ Pandya, ${ }^{7} \mathrm{~S} \mathrm{Au},{ }^{7} \mathrm{~S}$ Scollon, ${ }^{8} \mathrm{M}$ Adams, ${ }^{8} \mathrm{~N}$ Street, ${ }^{8}$ the Muscular Dystrophy Surveillance, Tracking, Research Network (MD STARnet). 'The University of lowa, lowa City, lowa, USA; ${ }^{2}$ New York State Department of Health, Troy, New York, USA; ${ }^{3}$ University of Arizona, Tucson, Arizona, USA; ${ }^{4}$ University of Rochester, Rochester, New York, USA; ${ }^{5}$ University of Colorado, Denver, Colorado, USA; ${ }^{6}$ Colorado Department of Public Health and Environment, Denver, Colorado, USA; ${ }^{7}$ Hawaii Department of Health, Honolulu, Hawaii, USA; ${ }^{8}$ Centers for Disease Control and Prevention, Atlanta, Georgia, USA

Introduction DBMD has an estimated prevalence of 1/3500 male births. Worldwide, this estimate varies, likely due to differences in diagnostic criteria, ascertainment, and survival. To date, no U.S. population-based DBMD prevalence data by race/ethnicity have been published.

Methods In 2002, the Centers for Disease Control and Prevention established the MD STARnet to conduct population-based DBMD surveillance in four U.S. sites. Each site conducts active surveillance to identify males with DBMD born since January 1982 . Using these data, we calculated DBMD prevalence by race/ethnic subgroups and birth intervals (1986-1990; 1991-1995; 1996-2000). Prevalence was calculated as: [number of DBMD males age 5-9 years/number of male residents, age 5-9 years]. With the average age at DBMD 